\title{
Evaluation of Stenting for Intracranial Original Article Internal Carotid Artery Stenosis Using Flow Reversal
}

Taku Matsuda, Koichi Satoh, Mami Hanaoka, Manabu Ishihara, and Kazuhito Matsuzaki

Objective: The safety of stenting for intracranial internal carotid artery stenosis using flow reversal embolic protection was evaluated.

Subjects and Methods: Of the 13 patients who underwent stenting between April 2011 and September 2016, those treated with (group F) and without (group N) flow reversal were compared retrospectively.

Results: An embolic complication was observed in one patient in the group $\mathrm{N}$ but none in the group $\mathrm{F}$. The diffusionweighted imaging (DWI)-positive rate was $0 \%$ in the $\mathrm{F}$ group.

Conclusion: Stenting for intracranial internal carotid artery stenosis using flow reversal is a safe and effective procedure.

Keywords > intracranial internal carotid artery stenosis, stenting, flow reversal

\section{Introduction}

The recurrence rate of symptomatic intracranial internal carotid artery stenosis is known to be high with antithrombotic therapy alone, ${ }^{1,2,3)}$ and intracranial stenting was expected to be effective for its treatment, but its effectiveness was rejected by a randomized clinical trial. ${ }^{4)}$ Presently, stenting for intracranial arterial stenosis is regarded as a treatment worth considering only when symptoms appear despite internal treatment and exert serious effects on hemodynamics according to the Japanese guideline for

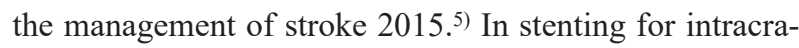
nial internal carotid artery stenosis, avoidance of hemorrhagic or embolic complications is important to obtain a favorable outcome. In this study, we evaluated stenting with flow reversal, which we perform as a treatment for intracranial internal carotid artery stenosis.

Division of Neurosurgery, Tokushima Red Cross Hospital, Komatsushima, Tokushima, Japan

Received: August 3, 2017; Accepted: October 13, 2017

Corresponding author: Taku Matsuda. Division of Neurosurgery, Tokushima Red Cross Hospital, 103 Irinokuchi, Komatsushimacho, Komatsushima, Tokushima 773-8502, Japan

Email: matsutaku.tokushima.osaka@gmail.com

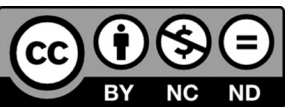

This work is licensed under a Creative Commons Attribution-NonCommercialNoDerivatives International License.

(C)2018 The Japanese Society for Neuroendovascular Therapy

\section{Subjects and Methods}

The subjects were 13 patients with $70 \%$ or severer stenosis of the intracranial internal carotid artery who underwent endovascular treatment (angioplasty and stenting) between April 2011 and September 2016. The 13 patients were divided into four early cases treated with no flow reversal (with no protection or with proximal protection: group $\mathrm{N}$ ) and nine late cases treated with flow reversal (group F), and the postprocedural technical success rate, stroke rate, and diffusion-weighted imaging (DWI)positive rate were compared. The preprocedural condition of the site of stenosis was evaluated by DSA, and dualantiplatelet therapy (aspirin at $100 \mathrm{mg} /$ day, clopidogrel at $75 \mathrm{mg} /$ day) was performed for at least 1 week before the procedure. In both groups, after placing a $5 \mathrm{Fr}$. sheath in the femoral artery under local or general anesthesia, DSA was performed using a $5 \mathrm{Fr}$. diagnostic catheter, and the site of stenosis, stenosis rate, and lesion length were measured. After replacing the sheath with a 6 Fr. or 9 Fr. device, systemic heparinization was performed, and the intraprocedural activated clotting time (ACT) was maintained at 250 seconds or longer.

In the group N, 6 Fr. Chaperon (Terumo Corporation, Tokyo, Japan) or 9 Fr. OPTIMO (Tokai Medical Products, Aichi, Japan) was placed in the internal carotid artery as a guiding catheter. Lesion cross was made using a microguidewire, the lesion was dilated using Gateway (Stryker, 


$\begin{array}{lcc}\text { Table } 1 & \begin{array}{l}\text { Baseline data between non flow reversal group (N) and flow reversal } \\ \text { group (F) }\end{array} & \\ \text { Characteristic } & \mathrm{N}(\mathrm{n}=4) & \mathrm{F}(\mathrm{n}=9) \\ \text { Age, mean (range) years } & 63.3(54-76) & 69.6(61-82) \\ \text { Male sex (\%) } & 4(100) & 6(66.7) \\ \text { Symptom } & 3(75) & 2(22) \\ \text { MnS (\%) } & 0(0) & 2(22) \\ \text { TIA (\%) } & 0(0) & 1(11) \\ \text { Tinnitus, no neurologic deficit (\%) } & 1(25) & 4(45) \\ \text { Asymptomatic (\%) } & 1(25) & 0(0) \\ \text { Location } & 1(25) & 1(11) \\ \text { C3 (\%) } & 1(25) & 1(11) \\ \text { C4 (\%) } & 1(25) & 7(78) \\ \text { C5 (\%) } & & \\ \text { Petrous (\%) } & 4(100) & 1(11) \\ \text { Anesthesia } & 0(0) & 8(89) \\ \text { Local (\%) } & 90(85-90) & 84.3(70-99) \\ \text { General (\%) } & 1(25) & 0(0) \\ \text { Stenosis, mean (range, \%) } & 3(75) & 9(100) \\ \text { Guiding Catheter } & \\ \text { 6 Fr. GC } & & \\ \text { 9 Fr. DLBC } & \end{array}$

DLBC: double lumen balloon catheter; GC: guiding catheter; MnS: minor stroke; TIA: transient ischemic attack

Fremont, CA, USA), and Integrity (Medtronic, Minneapolis, MN, USA) or Driver (Medtronic), which are coronary stent systems, was placed. Predilatation and stenting were performed without protection if $6 \mathrm{Fr}$. Chaperon was used as the guiding catheter and with occlusion by inflating the balloon if 9 Fr. OPTIMO was used. Then, $50-100 \mathrm{~mL}$ of blood was manually aspirated, and, after confirming the absence of floating thrombi, occlusion was released.

In the group F, a $5 \mathrm{Fr}$. sheath was also placed in the femoral vein, 9 Fr. OPTIMO was placed in the internal carotid artery as a guiding catheter, connected with the sheath in the femoral vein via a $\mathrm{Y}$ connector of the $9 \mathrm{Fr}$. OPTIMO by interposing a transfusion filter before balloon occlusion, and a complete flow reversal condition was prepared. Lesion cross was made using a microguidewire, the site of stenosis was predilated using Gateway (Stryker), and Integrity or Wingspan stent (Stryker) was placed at the site of stenosis.

In both groups, MRI was performed on the day after the procedure, and the presence or absence of new foci of cerebral infarction was checked by DWI. As a follow-up, the stenting site was examined by 3D-CTA or DSA in addition to neurologic evaluation after 3 months.

\section{Results}

Table 1 shows the details of each group. The mean age was 63.3 and 69.6 years in the groups $\mathrm{N}$ and F, respectively. The onset pattern was minor stroke $(\mathrm{MnS})$ in three patients
$(75 \%)$ in the group $\mathrm{N}$ and two patients (22\%) in the group $\mathrm{F}$ but one patient $(25 \%)$ in group $\mathrm{N}$ and four patients $(45 \%)$ in group $\mathrm{F}$ were asymptomatic; the frequency of $\mathrm{MnS}$ was high in the group $\mathrm{N}$. The site of stenosis was the petrous portion in $78 \%$ in the group F. Anesthesia was local in all patients in the group $\mathrm{N}$ but general in all but the first patient in the group $\mathrm{F}$ in consideration of the possibility of occlusion intolerance. The length of stenosis was $5-10 \mathrm{~mm}$ in all patients (Mori Type B) with no difference between the two groups. Table 2 shows the devices used and complications in individual patients. Two representative cases, i.e., Case 4 that suffered an embolic complication and Case 10 that underwent stenting with flow reversal, are presented.

Case 4 was a 59-year-old male (Fig. 1) who had undergone carotid artery stenting (CAS) for right cervical carotid artery stenosis at another hospital. He consulted a local doctor due to amaurosis fugax and was found to have stenosis of the left intracranial internal carotid artery. DSA confirmed $85 \%$ stenosis in the petrous segment of the left internal carotid artery (Fig. 1a), and stenting was performed. The internal carotid artery was occluded with $9 \mathrm{Fr}$. OPTIMO, and after predilatation using Gateway, Integrity was placed. Occlusion was released after manually aspirating about $100 \mathrm{~mL}$ of blood, and angiography showed improvement of the stenosis rate to nearly $0 \%$ (Fig. 1 $\mathbf{1 b}$ and 1c). However, dysarthria and right hemiparesis appeared immediately after the release of occlusion. MRI immediately after the procedure showed new diffuse 
Table 2 Characteristics, devices, and complications of all patients

\begin{tabular}{|c|c|c|c|c|c|c|c|c|c|c|}
\hline Group & Case & Age & Sex & Site & Stenosis (\%) & Symptom & Stent & $\mathrm{GC}$ & Anesthesia & Complication \\
\hline $\mathrm{N}$ & 1 & 65 & $\mathrm{M}$ & Rt. C3 & 90 & Asymptomatic & CS & 9 Fr. DLBC & Local & - \\
\hline $\mathrm{N}$ & 2 & 54 & $\mathrm{M}$ & Rt. C4 & 95 & $\mathrm{MnS}$ & CS & 6 Fr. GC & Local & - \\
\hline $\mathrm{N}$ & 3 & 76 & $\mathrm{M}$ & Rt. C5 & 76 & $\mathrm{MnS}$ & CS & 9 Fr. DLBC & Local & - \\
\hline $\mathrm{N}$ & 4 & 59 & $\mathrm{M}$ & Lt. P & 85 & $\mathrm{MnS}$ & CS & 9 Fr. DLBC & Local & Shower emboli \\
\hline$F$ & 5 & 71 & $\mathrm{M}$ & Rt. P & 85 & TIA & CS & 9 Fr. DLBC & Local & \\
\hline $\mathrm{F}$ & 6 & 82 & $\mathrm{M}$ & Rt. P & 85 & Asymptomatic & CS & 9 Fr. DLBC & General & - \\
\hline $\mathrm{F}$ & 7 & 68 & $\mathrm{M}$ & Rt. P & 90 & Asymptomatic & $\mathrm{CS}$ & 9 Fr. DLBC & General & - \\
\hline $\mathrm{F}$ & 8 & 61 & $\mathrm{~F}$ & Rt. P & 85 & $\mathrm{MnS}$ & WS & 9 Fr. DLBC & General & - \\
\hline $\mathrm{F}$ & 9 & 75 & $\mathrm{~F}$ & Rt. P & 75 & Asymptomatic & CS & 9 Fr. DLBC & General & - \\
\hline $\mathrm{F}$ & 10 & 65 & $\mathrm{M}$ & Lt. P & 90 & $\mathrm{MnS}$ & CS, WS & 9 Fr. DLBC & General & - \\
\hline $\mathrm{F}$ & 11 & 67 & $\mathrm{M}$ & Rt. C5 & 80 & Asymptomatic & CS & 9 Fr. DLBC & General & - \\
\hline $\mathrm{F}$ & 12 & 63 & $\mathrm{~F}$ & Rt. C4 & 70 & Tinnitus & WS & 9 Fr. DLBC & General & - \\
\hline $\mathrm{F}$ & 13 & 74 & M & Rt. P & 99 & TIA & CS & 9 Fr. DLBC & General & - \\
\hline
\end{tabular}

CS: coronary stent; DLBC: double lumen balloon catheter; F: flow reversal group; GC: guiding catheter; Lt: left; MnS: minor stroke; N: non flow reversal group; P: petrous portion; Rt: right; TIA: transient ischemic stroke; WS: wingspan stent
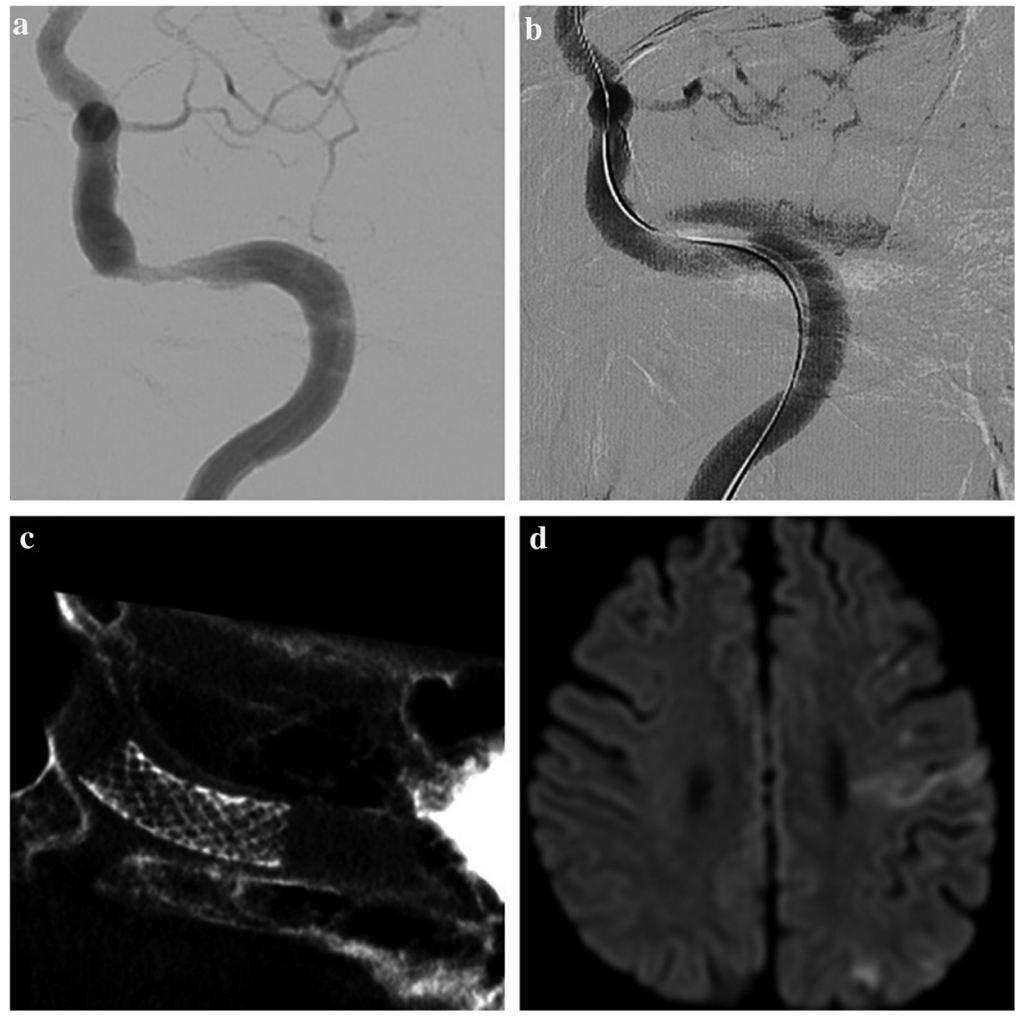

Fig. 1 A 59-year-old man with amaurosis fugax due to stenosis of the left intracranial internal carotid artery. (a) Anteroposterior view of left carotid angiography shows $85 \%$ stenosis of left intracranial internal carotid artery petrous portion. (b) Just after stenting, angiography shows complete recanalization without residual stenosis. (c) Cone-beam CT shows good expansion of the stent. (d) Axial DWI after stenting shows new ischemic lesions in the left hemisphere. DWI: diffusionweighted imaging

hyperintensities in the left middle cerebral artery territory (Fig. 1d). With dysarthria and right hemiparesis persisting, the patient was transferred to a rehabilitation hospital on the 10th postprocedural day with a modified Rankin scale (mRS) score of 2.
Case 10 was a 65-year-old male (Fig. 2) who consulted the outpatient clinic due to dizziness and dysarthria. MRI showed a focus of subacute infarction in the left parietal lobe (Fig. 2a), and DSA showed $90 \%$ stenosis of the petrous segment of the left internal carotid artery (Fig. 2b). 

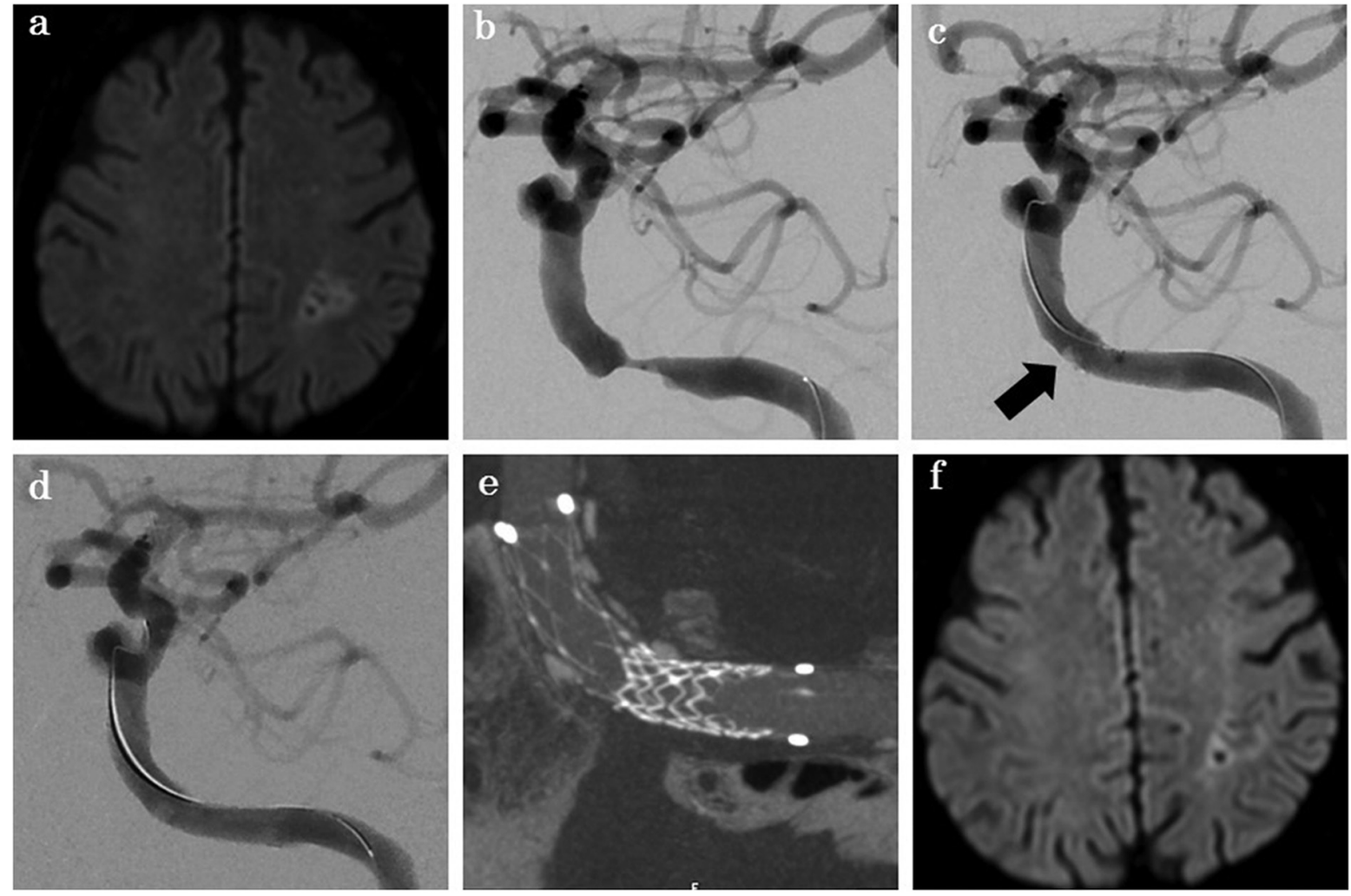

Fig. 2 A 65-year-old man with a left subacute infarction due to stenosis of the intracranial internal carotid artery stenosis. (a) Axial DW on admission shows subacute infarction in the left parietal lobe. (b) Anteroposterior view of left carotid angiography shows $90 \%$ stenosis of left intracranial internal carotid artery petrous portion. (c) Just after stenting, angiography shows partial recanalization with plaque protrusion (arrow). (d) After stent in stent (Wingspan stent; Stryker, Fremont, CA, USA), angiography shows complete recanalization without residual stenosis. (e) Cone-beam CT shows good expansion of the double stents. (f) Axial DWI after stenting shows no new ischemic lesion. DWI: diffusion-weighted imaging

Table 3 Postprocedural complications between non flow reversal $(\mathrm{N})$ and flow reversal group $(\mathrm{F})$

\begin{tabular}{lcl} 
& $N(n=4)$ & $F(n=9)$ \\
Technical success rate (\%) & $4(100)$ & $9(100)$ \\
Stroke rate (\%) & $1(25)$ & $0(0)$ \\
DWI positive (\%) & $1(25)$ & $0(0)$ \\
\hline
\end{tabular}

DWl: diffusion-weighted imaging

After 3 weeks, stenting was performed with flow reversal. In a flow-reversed state, Integrity was placed after predilatation using Gateway. Since plaque shift was noted after stent placement (Fig. 2c), blood flow was reversed again, and an additional Wingspan stent was placed, resulting in an improvement of the stenosis rate to nearly $0 \%$ (Fig. 2d and 2e). There was no change in neurologic symptoms after the procedure, no new foci of cerebral infarction were noted on MRI after 3 days (Fig. 2f), and the patient was discharged to home 7 days after admission.

The technical success rate was $100 \%$ in both groups, but the stroke rate was $25 \%$, and the DWI-positive rate was $25 \%$, in the group N. In the group F, no complication was noted, and the stroke rate and DWI-positive rate were both $0 \%$ (Table 3 ).

\section{Discussion}

The recurrence rate of intracranial internal artery stenosis is known to be high after medical treatment with antiplatelet agents. ${ }^{1,2,3)}$ Angioplasty using a balloon catheter for intracranial arterial stenosis began in the 1990s. Mori et al. classified lesions into three types (type A, short concentric; type B, tubular extremely eccentric; and type C, diffuse) and reported the long-term prognosis after balloon angioplasty. In type A, effective dilatation could be obtained with low risk, and a relatively favorable long-term outcome could be expected. In types B and C, however, the risk was high, and effective dilatation could not be achieved in many patients. $\left.{ }^{6}\right)$ To overcome such problems with the balloon catheter, metal stents were introduced with a high expectation, and their effectiveness and favorable therapeutic results have been reported. ${ }^{7,8)}$ We used coronary 
artery stents in 11 of our 13 patients, but the application of balloon expandable stents for the coronary artery to intracranial vessels is limited to particular sites such as non-curved and lesions with low volume plaque.

Under such circumstances, great expectations were directed to the Wingspan, which is a self-expandable stent, but many periprocedural ischemic complications were reported in a randomized clinical trial, and the outcomes were clearly inferior to those of medical treatment. ${ }^{4)}$ The poor outcome may be explained by occlusion of perforating arteries and distal embolism. In stenting for the intracranial internal carotid artery stenosis, in which the amount of plaque is smaller than in stenosis of the origin of the carotid artery, occlusion of perforating arteries tends to be regarded as a greater concern than distal embolism. Indeed, three-fourths of the lesions had many perforating arteries in the SAMMPRIS study, and their occlusion may have contributed to the increase in ischemic complications. Regarding distal embolic protection in stenting for intracranial internal carotid artery stenosis, there is a report that complications due to distal embolism were rare even without special protection such as proximal flow control, ${ }^{9}$ on the contrary, there is a report recommending aggressive proximal protection. ${ }^{10)}$ In our Case 4, obvious shower emboli were caused despite implementation of proximal protection, and proximal protection alone was suggested to be insufficient to prevent distal embolism due to plaque rupture. After experiencing this case, we performed stenting with flow reversal.

In cervical CAS, devices for the prevention of distal embolism have been developed with time. Satoh et al. reported that the DWI-positive rate after CAS was 64\% when the filter type ANGIOGUARD XP (Cordis, Milpitas, CA, USA) was used but was reduced to $17.8 \%$ by combining distal occlusion using PercuSurge GuardWire (Medtronic) with blood aspiration. ${ }^{11)}$ In addition, the effectiveness of the Parodi antiembolic system in CAS has been demonstrated, and there is a report that the incidence of stroke or death within 30 days after treatment could be reduced to $1.5 \%$ by this method. ${ }^{12)}$ Also, Asakura et al. reported that the DWI-positive rate after CAS using the Parodi antiembolic system was $18.2 \%$ and did not differ significantly compared with $11.5 \%$ using diagnostic angiography. ${ }^{13)}$

In CAS, complete flow reversal may not be implemented with the Parodi antiembolic system due to the presence of the superior thyroid artery. However, complete flow reversal can be achieved in intracranial internal carotid artery stenosis in majority of cases. In the patients reported here, the DWI-positive rate after stenting with flow reversal was $0 \%$, indicating a high preventive effect against distal embolism.

\section{Conclusion}

Stenting with flow reversal is likely to be an effective and safe treatment for intracranial internal carotid artery stenosis.

\section{Disclosure Statement}

Neither the first author nor any of the coauthors have any conflicts of interest to declare.

\section{References}

1) Gorelick PB, Wong KS, Bae HJ, et al: Large artery intracranial occlusive disease: a large worldwide burden but a relatively neglected frontier. Stroke 2008; 39: 2396-2399.

2) Wong KS, Li H: Long-term mortality and recurrent stroke risk among Chinese stroke patients with predominant intracranial atherosclerosis. Stroke 2003; 34: 2361-2366.

3) Kasner SE, Chimowitz MI, Lynn MJ, et al: Predictors of ischemic stroke in the territory of a symptomatic intracranial arterial stenosis. Circulation 2006; 113: 555-563.

4) Chimowitz MI, Lynn MJ, Derdeyn CP, et al: Stenting versus aggressive medical therapy for intracranial arterial stenosis. N Engl J Med 2011; 365: 993-1003.

5) Terada T, Sakai N, Sugiu K: [Intracranial arterial stenosis. In: The clinical guideline of Neuroendovascular Therapy]. JNET 2009; 3(Supple. 1): 66-70. (in Japanese)

6) Mori T, Fukuoka M, Kazita K, et al: Follow-up study after intracranial percutaneous transluminal cerebral balloon angioplasty. AJNR Am J Neuroradiol 1998; 19: 1525-1533.

7) Kessler IM, Mounayer C, Piotin M, et al: The use of balloon-expandable stents in the management of intracranial arterial diseases: a 5-year single-center experience. AJNR Am J Neuroradiol 2005; 26: 2342-2348.

8) Terada T, Tsuura M, Matsumoto H, et al: Endovascular therapy for stenosis of the petrous or cavernous portion of the internal carotid artery: percutaneous transluminal angioplasty compared with stent placement. J Neurosurg 2003; 98: 491-497.

9) Tsumoto $T$, Terada $T$, Tsuura M, et al: Diffusion-weighted imaging abnormalities after percutaneous transluminal angioplasty and stenting for intracranial atherosclerotic disease. AJNR Am J Neuroradiol 2005; 26: 385-389. 
10) Kondoh R, Furui E, Matsumoto Y: [Stenting of symptomatic intracranial atherosclerotic stenosis]. JNET 2008; 2: 201-206. (in Japanese)

11) Satoh K, Hanaoka M, Tamura T, et al: [Transition of carotid artery stenting (CAS) treatment in the multiple device era (effectiveness of distal balloon protection CAS treatment using an autologous aspirated blood transfusion)]. Surg Cereb Stroke 2015; 43: 347-351. (in Japanese)
12) Parodi JC, Schönholz C, Parodi FE, et al: Initial 200 cases of carotid artery stenting using a reversal-of-flow cerebral protection device. J Cardiovasc Surg 2007; 48: 117-124.

13) Asakura F, Kawaguchi K, Sakaida H, et al: Diffusionweighted MR imaging in carotid angioplasty and stenting with protection by the reversed carotid arterial flow. AJNR Am J Neuroradiol 2006; 27: 753-758. 